\title{
Dissociating Controlled from Automatic Processing in Temporal Preparation
}

\author{
Mariagrazia Capizzi, Daniel Sanabria and Ángel Correa \\ Departamento de Psicología Experimental \\ Universidad de Granada, Spain
}

Keywords: temporal orienting, sequential effects, dual-task, working memory.

\section{Highlights:}

$>$ Automaticity of temporal orienting and sequential effects was tested by dual-task methodology $>$ Sequential effects were not attenuated by concurrent dual-task demands $>$ Temporal orienting was reduced by competition for executive resources required by a concurrent working memory task $>$ Results dissociated the involvement of automatic and controlled processes in temporal preparation.

Date: $16-01-2012$

NOTICE: This is the authors' version of a work that was accepted for publication in Cognition, and can be used for scholarly non-commercial purposes. Changes resulting from the publishing process, such as editing, corrections, structural formatting, and other quality control mechanisms may not be reflected in this document. Definitive version will be subsequently published in Cognition.

Correspondence regarding this article should be directed to: Mariagrazia Capizzi, Departamento de Psicología Experimental, Campus de Cartuja, s/n, Universidad de Granada, 18071, Spain. E-mail: mgcapizzi@ugr.es. 


\begin{abstract}
The aim of the present study was to investigate the controlled versus the automatic nature of temporal preparation. If temporal preparation involves controlled rather than automatic processing, it should be reduced by the addition of a concurrent demanding task. This hypothesis was tested by comparing participants' performance in a temporal orienting task that measured two main effects of temporal preparation (temporal orienting and sequential effects) between a single-task and a dual-task condition. In the single-task condition, participants responded to a visual target presented after symbolic cues that were highly predictive of the moment of target onset. In the dual-task condition, the temporal preparation task was performed concurrently with a working memory task. The results showed that sequential effects survived to dual-task interference, while temporal orienting was reduced as a function of the competition for executive resources required by both working memory and temporal preparation tasks. These findings provide direct behavioural evidence that temporal orienting and sequential effects involve dissociable cognitive processes.
\end{abstract}




\section{Introduction}

The ability to anticipate and prepare an optimized response to forthcoming events, i.e. temporal preparation, is essential to many forms of cognitive and motor behaviour (Nobre, Correa, \& Coull, 2007). Temporal preparation can be flexibly built up on the basis of different information, such as that provided by temporal predictions (temporal orienting effects) and duration of the preceding time interval (sequential effects). An ongoing debate in the literature is to what extent these temporal preparation effects are mediated by controlled or automatic processing. To address this issue, in the present study we used a dual-task paradigm in which a temporal preparation task had to be performed concurrently with a working memory (WM) task. Our aim was to test the nature of the processes involved in these two main temporal preparation effects by investigating whether they would survive or suffer interference from a concurrent secondary task.

One of the most used experimental procedures to measure temporal preparation is the temporal orienting task (Coull and Nobre, 1998; see Nobre, 2001, for a review). In this paradigm, which was modelled after Posner's spatial orienting task (Posner, Snyder, \& Davidson, 1980), a symbolic cue predicts with high probability the specific time interval (i.e., early versus late) at which the target stimulus would occur. Temporal orienting effects are typically revealed by faster and/or more accurate responses to targets appearing at early validly cued temporal intervals as compared to earlier than expected targets. At the long time interval, temporal orienting effects are usually smaller or even absent. A widely accepted proposal put forward by some authors (Correa, Lupiáñez, Milliken, \& Tudela, 2004; Coull \& Nobre, 1998; Karlin, 1959) to explain the attenuation of temporal orienting effects at the long interval is based on the 
"reorienting of attention". According to it, if the target does not appear early as predicted, participants infer that it will appear later, which enables them to reorient their attention accordingly.

Another reliable finding in temporal preparation research concerns the influence of the previous time interval duration on performance in the current trial. Participants' RT is typically faster when a short interval is preceded by another short rather than long interval, i.e., sequential effects (Drazin, 1961; Los \& Van den Heuvel, 2001; Steinborn, Rolke, Bratzke, \& Ulrich, 2008; Vallesi \& Shallice, 2007; Woodrow, 1914). Sequential effects are usually asymmetric since at the long time interval participants' RT is equally fast when the previous interval was either short or long.

It has been suggested that sequential effects are 'automatic', reflecting the involvement of processes exogenously triggered by stimulus sequence association from one trial to the next, rather than by internal volitional expectations (see Los' trace conditioning account for further details, Los, 1996; Los \& Heslenfeld, 2005; Los \& Van den Heuvel, 2001). In support of the automaticity of sequential effects, behavioural and electrophysiological studies have found dissociations between temporal orienting and sequential effects (Correa et al., 2004; Correa, Lupiáñez, \& Tudela, 2006; Los \& Heslenfeld, 2005; Los \& Van den Heuvel, 2001). For example, Los and Van den Heuvel (2001) first demonstrated that sequential effects are stronger in the absence of temporal orienting effects, after invalid temporal cues, than in the presence of temporal orienting, after valid temporal cues. More recent neuropsychological research has shown that patients with prefrontal lesions exhibit intact sequential effects, despite reduced temporal orienting effects (Triviño et al., 2010). Triviño et al.’s study (2010) suggests that different temporal preparation processes might underlie the dissociation 
between temporal orienting and sequential effects. That is, temporal orienting effects would involve controlled processing as they would depend on the functioning of a typical structure of attentional control (i.e., the prefrontal cortex), while sequential effects would be the result of automatic processing as they would not require the involvement of the prefrontal cortex. However, this neural criterion could be questioned since it only supports the distinction between automatic and controlled temporal preparation processing rather indirectly. It remains to be determined the extent to which temporal preparation effects are accomplished by automatic or controlled processing. To our knowledge, no study has yet tested directly the automaticity of both temporal orienting and sequential effects in a single experimental design (but see Van Lambagen \& Los, 2008, for a dual-task study on sequential effects). Therefore, our goal was to explore the nature of the processes involved in these two main temporal preparation effects by comparing how they would behave in a dual-task context.

In a typical dual-task experiment, two tasks (commonly called the primary and the secondary task) are performed simultaneously. Assuming that primary and secondary tasks compete for common limited resources, the addition of a secondary task should interfere with performance on the primary task (e.g., Logan, 1979). Thus, the key criterion in our study to dissociate controlled from automatic temporal preparation was that the primary task involves controlled processing when the addition of a concurrent task would impair behaviour by decreasing participants' performance, whereas the primary task involves automatic processing when performance would not be diminished by the secondary task (Logan, 1978, 1979; Posner \& Snyder, 1975). In our dual-task study, the primary task was a simple-RT task that measured temporal preparation. Participants had to respond to a visual target that was preceded by a 
symbolic cue (a short versus a long line) and that appeared after one of two different time intervals in the seconds range ( $1 \mathrm{~s}$ versus $3 \mathrm{~s})$. The secondary task demanded WM, i.e. under dual-task conditions participants performed a mental counting task in which they continuously updated information held in WM.

We decided to use a WM task as secondary task on the basis of the following evidence. First, previous dual-task studies have shown interference between WM and time estimation of intervals in the range of seconds, suggesting that these two tasks may draw on the same cognitive resources for executive control (e.g., Brown, 2006; Fortin \& Breton, 1995). Second, both WM and timing tasks have been shown to engage prefrontal structures (see Lewis \& Miall, 2006, for a review), which likewise relate to temporal orienting effects (Coull \& Nobre, 1998; Triviño et al., 2010). According to the above findings, we reasoned that if temporal orienting effects rely on controlled processing, they should be reduced by the addition of a demanding secondary task. By contrast, if sequential effects are automatically elicited by stimulus sequence association, they should not be attenuated by the addition of a concurrent task.

\section{EXPERIMENT 1}

In Experiment 1, the primary task was a simple-RT task in which temporal expectancy was manipulated between blocks of trials to optimize the finding of attentional effects (Correa et al., 2006). The secondary task of the dual-task condition required concurrently updating the count of different colours that the temporal cues displayed on every trial and reporting the final count at the end of each block. Based on 
a previous study showing reduced temporal orienting effects after prefrontal damage (Triviño et al., 2010), we expected temporal orienting, but not sequential effects, to be attenuated by competing WM demands in the dual-task condition as compared to the single-task condition.

\section{Method}

Participants. Fifty-nine undergraduates from the University of Granada (forty-seven females, five left-handed, age range: 18-34 years old) took part in Experiment 1. Data from one participant were excluded from analysis due to missing observations in one experimental condition. All the participants in all the experiments reported in this article had normal or corrected-to-normal vision, none of them was colour-blind, and all received course credits for their participation. The study was conducted in accordance with the ethical standards of the1964 Declaration of Helsinki.

Apparatus and stimuli. The experiment was run on an Intel Core 2 Duo personal computer connected to a 17 " LCD monitor. Stimulus presentation and data recording were controlled by E-prime software (Schneider, Eschman, \& Zuccolotto, 2002). The viewing distance was approximately $57 \mathrm{~cm}$. All stimuli were presented on a black background in the centre of the screen. The temporal cues consisted of a short line $\left(3.4^{\circ}\right.$ $\mathrm{x} 1.3^{\circ}$ visual angle) and a long line $\left(7.5^{\circ} \times 1.3^{\circ}\right)$ presented either in red, green or blue. The short line indicated that the target would probably appear early (after $1 \mathrm{~s}$ ) and the long line indicated that the target would probably appear late (after $3 \mathrm{~s}$ ). The target stimulus was a white dot (diameter: $\left.1.5^{\circ}\right)$. 
Procedure and Task. Participants were tested in a silent and dimly illuminated room. Both written and verbal instructions were provided for the single-task and the dual-task condition. A trial of the single-task block began with the presentation of a blank screen for a random duration between 500 and $1000 \mathrm{~ms}$ (see Figure 1). The temporal cue, filled with one of three colours (red, green, or blue), was then displayed for $750 \mathrm{~ms}$. Each colour was randomly generated at the beginning of each trial with the same probability of appearance. Participants were told that the colour of the temporal cue was taskirrelevant and should therefore ignore it. Following the cue, the screen remained blank for a variable delay of either 1 or $3 \mathrm{~s}$, depending on the time interval for that trial. After the time interval elapsed, the target stimulus was presented for $100 \mathrm{~ms}$ and then disappeared. Participants had to respond to the target onset as quickly as possible by pressing the spacebar with the index finger of their preferred hand. They were explicitly informed that the temporal cue would help them to predict the occurrence of the forthcoming target. A visual feedback message was displayed for $500 \mathrm{~ms}$ either in case a premature response was given before the onset of the target ("wait for the target") or if no response was made within $1100 \mathrm{~ms}$ after the offset of the target ("respond earlier"). Following the response to the target, or after $1100 \mathrm{~ms}$ in case of a missed response, the next trial began. 


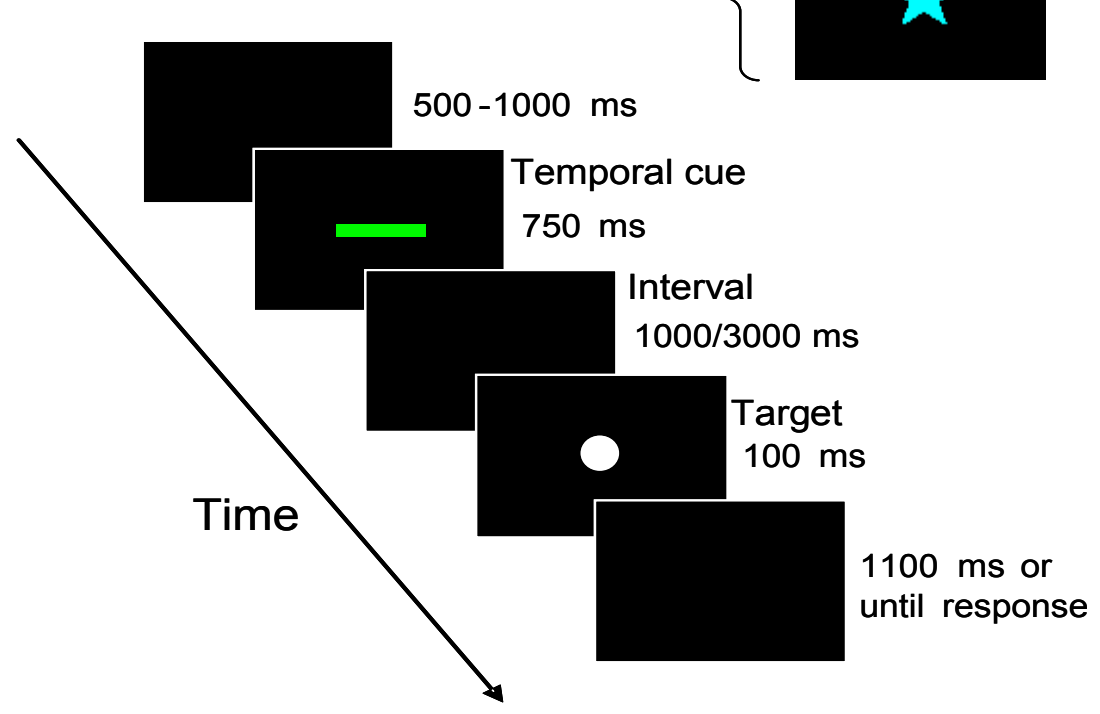

Figure 1. Schematic representation of events in a trial in Experiments 1 and 2. The only variation was that in Experiment 3 the temporal cue was presented in gray and the memory stimulus consisted of one of three coloured (red, green or blue) stars.

In the dual-task condition, the temporal preparation task remained the same as that described above. The only difference with respect to the single-task condition was the addition of the concurrent WM task. The WM task required participants to count and remember how many times each temporal cue colour appeared during a block of trials. At the end of the block, one of the three colours was randomly chosen (e.g., "red") and participants had to type how many times that colour had been presented. Each colour was equally probable to be selected for the memory test. This task 
encouraged participants to update their WM contents on every trial, in order to maintain the final count of each colour until the end of the block. Feedback about memory accuracy (the word "correct" or "incorrect" in white for $1500 \mathrm{~ms}$ ) was provided after the response in each block to engage participants in the WM task. Participants' instructions, however, emphasized equal priority to temporal preparation and WM tasks.

For each task condition, participants completed seven blocks of 16 trials each of one temporal cue (e.g., early), and seven blocks of the remaining temporal cue (late). The order of presentation of early and late cue blocks was counterbalanced across participants within each single-task and dual-task condition. Half of the participants began with the single-task condition, and half began with the dual-task condition. The first block of each task condition was considered as practice. For each block, the validity proportion was of .75 , i.e. 12 trials were valid trials in which the cue was early (or late) and the target appeared after the 1-s (or 3-s) time interval, whereas 4 trials were invalid, in which the cue was early (or late) and the target appeared after the 3-s (or 1-s) time interval. Participants received feedback on RT detection performance only during practice trials. A rest between blocks of trials was allowed. The whole session lasted about 45 minutes.

Design and data analysis. Experiment 1 constituted a within-participants design with the independent variables of Task (single-task versus dual-task), Validity (valid versus invalid), Previous interval (short versus long) and Current interval (short versus long). Participants' RT to respond to the target was the dependent variable. Temporal orienting effects were indexed by the main effect of Validity. Sequential effects were indexed by 
the main effect of Previous interval and their asymmetry by the interaction between Previous interval and Current interval.

Data from practice trials, the first trial of each block, trials with premature responses (i.e., responses before target onset: $2.4 \%$ ), trials with RT below $150 \mathrm{~ms}(0.3$ $\%)$ and above $1000 \mathrm{~ms}(0.2 \%)$, and trials without responses $(0.8 \%)$ were rejected from the analysis. Mean RTs for each participant and condition were analysed by a repeatedmeasures ANOVA.

Results

In the WM task, the overall accuracy across participants to the colour memory test was $0.7^{1}$.

In the temporal preparation task, the significant main effect of Task, $F(1,57)=$ $91.01, p<.001$, showed that participants were slower in the dual-task condition than in the single-task condition. The main effect of Validity was also significant, $F(1,57)=$ 59.74, $p<.001$, with faster RTs for valid trials than for invalid trials. There was a significant interaction between Validity and Current interval, $F(1,57)=37.72, p<.001$. In line with previous temporal orienting studies (Nobre, 2001), the Validity effect was larger at the short interval $(41 \mathrm{~ms})$ than at the long interval $(-7 \mathrm{~ms})$, although it reached significance in both time intervals $[F(1,57)=60.06, p<.001, F(1,57)=3.86, p=.05$, for the short and the long interval, respectively]. However, contrary to our prediction, the Validity effect was not modulated by Task condition $\left(F_{\mathbf{S}}<1\right.$ for both Task $\mathrm{x}$ Validity and Task x Validity x Current interval interactions; see Figure 2). 


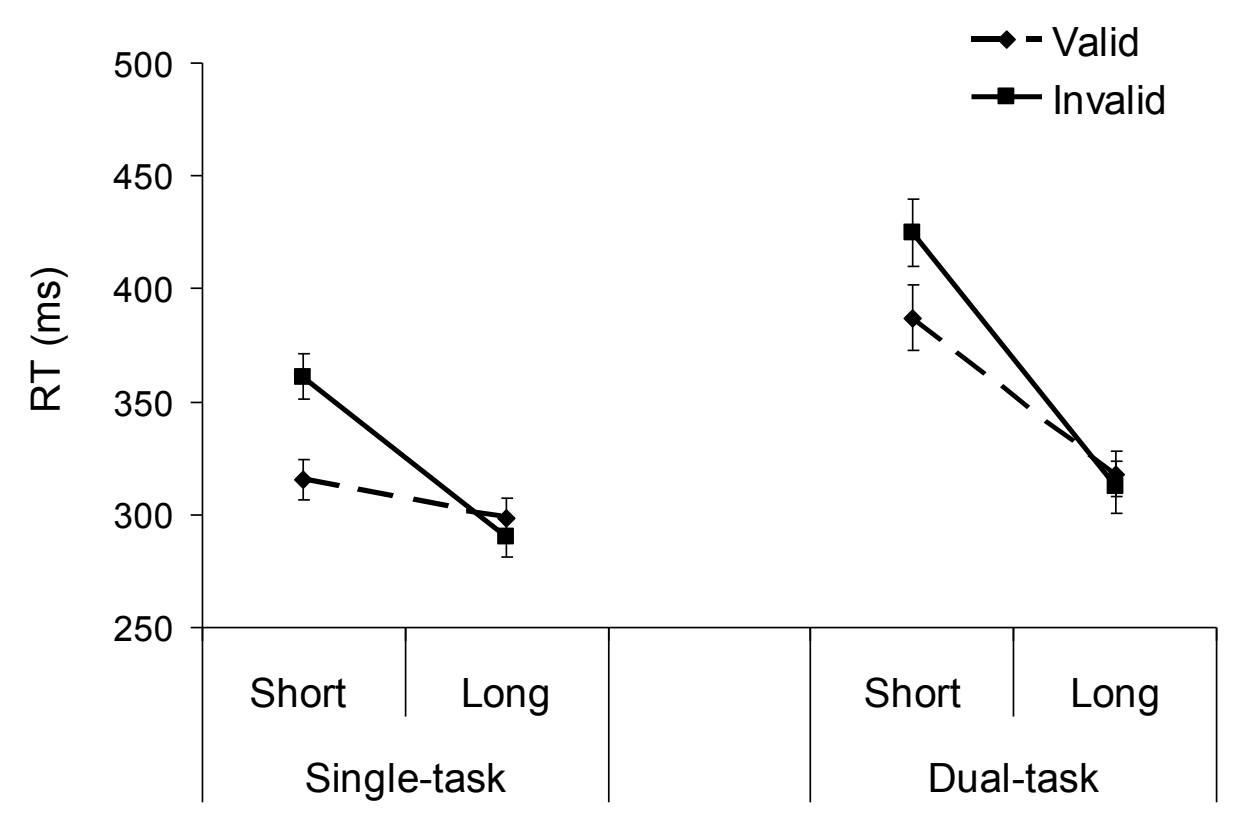

Figure 2. Mean RTs as a function of Task (single-task vs. dual-task), Validity (valid vs. invalid) and Current interval (short vs. long) for Experiment 1 (vertical bars represent standard error of the mean).

Regarding sequential effects, the main effect of Previous interval was significant, $F(1,57)=264.8, p<.001$, indicating that participants responded faster after a previous short interval than after a previous long interval. The significant interaction between Previous interval and Current interval, $F(1,57)=116.4, p<.001$, replicated the typical asymmetry of sequential effects, with a larger effect of the previous interval at the current short interval (53 ms) than at the current long interval $(12 \mathrm{~ms})$, although it reached significance in both time intervals $[F(1,57)=371.8, p<.001, F(1,57)=19.01, p$ $<.001$, for the short and the long interval, respectively]. Crucially, sequential effects 
were not modulated by Task condition (see Figure 3), since the interactions involving Task and Previous interval factors were not statistically significant $(F \mathrm{~s}<1$ for both Task $\mathrm{x}$ Previous interval and Task x Previous interval x Current interval interactions).

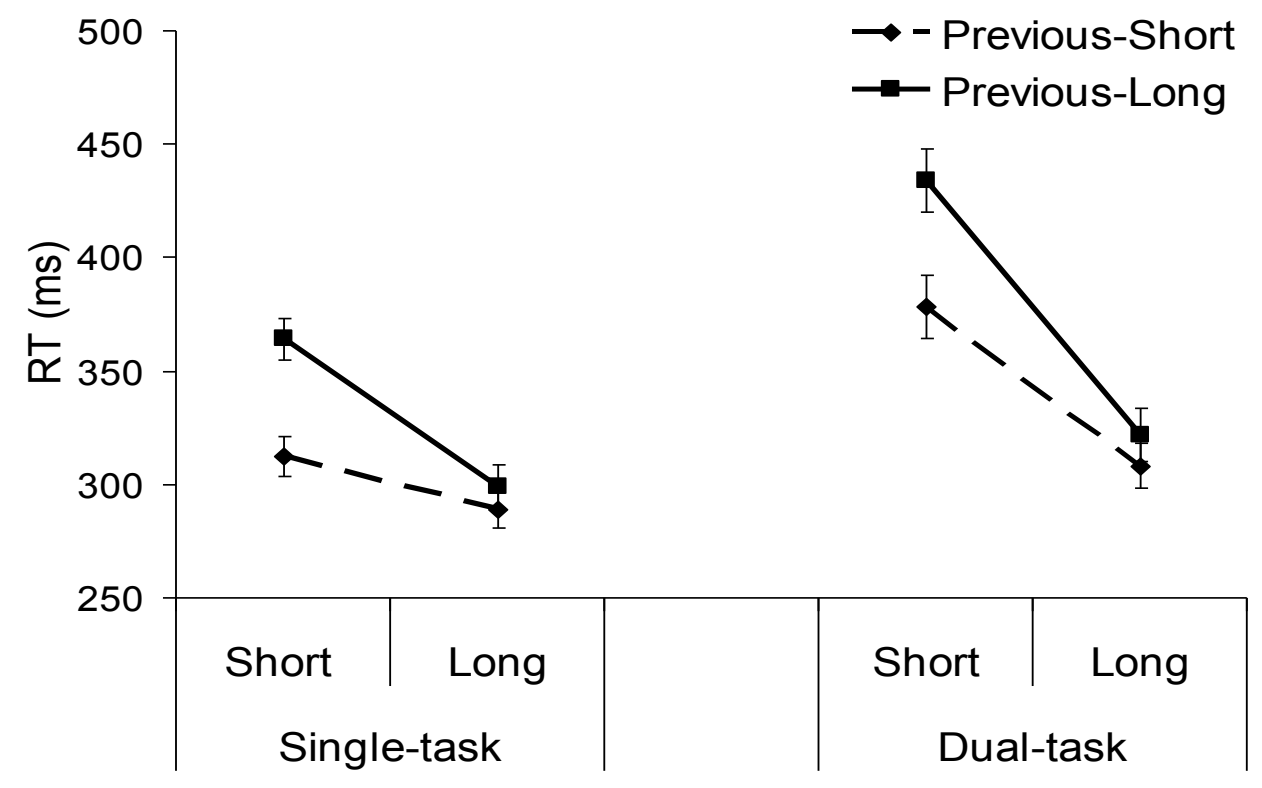

Figure 3. Mean RTs as a function of Task (single-task vs. dual-task), Previous interval (short vs. long) and Current interval (short vs. long) for Experiment 1 (vertical bars represent standard error of the mean).

Moreover, there was a significant main effect of Current interval, $F(1,57)=$ $281.5, p<.001$, with participants responding faster at the long time interval as compared to the short time interval. The interaction between Task and Current interval was also significant, $F(1,57)=75.23, p<.001$. Further comparisons for this interaction ${ }^{2}$ showed 
a greater difference in participants' RT between the single-task and the dual-task condition at the short interval (68 ms) as compared to the long interval (21 ms), with the task effect reaching statistical significance in both time intervals $[F(1,57)=120.6, p<$ .001 and $F(1,57)=22.22, p<.001$, for the short and the long interval, respectively]. None of the remaining terms of the ANOVA reached statistical significance.

\section{Discussion}

The results of Experiment 1 showed a significant difference in temporal preparation performance between the single-task and the dual-task condition: participants' response time to the target was impaired by the concurrent colour counting task. This finding confirmed that our manipulation of WM was effective and that temporal preparation is sensitive to extra demands of executive control. Unexpectedly, however, both temporal orienting and sequential effects survived to dual-task interference. In fact, contrary to our prediction on temporal orienting, the validity effect was of similar magnitude under both the single-task and the dual-task condition.

As temporal cues were manipulated in a blocked design, it is possible that temporal orienting was contaminated by the presence of strong sequential effects. That is, the early block and the late block could be biased by the high frequency of short and long intertrial sequences, respectively, so that the final shape of cueing effects would stem from sequential transitions over successive trials rather than from temporal orienting. This would account for the lack of dual-task interference on both temporal orienting and sequential effects. To explore this possibility, we made a reanalysis of the data of Experiment 1 with the factors of Task, Temporal expectancy, Previous interval and Current interval, which revealed a significant interaction between Temporal 
expectancy and Current interval, $F(1,57)=59.74, p<.001$ (i.e., temporal orienting effects) that, crucially, was independent of sequential effects (Temporal expectancy $\mathrm{x}$ Previous interval x Current interval: $F<1$ ). This result replicates the findings by Correa et al. (2004, 2006), suggesting that the contribution of temporal orienting is independent from the presence of sequential effects in the context of a blocked-manipulation of temporal expectancies.

A more plausible explanation for the finding that temporal orienting survived to dual-task interference considers that the cueing manipulation employed in this experiment was confounded with a foreperiod (i.e., the time interval between the cue and the target) distribution effect. Note that the early block contained $75 \%$ of short foreperiods and $25 \%$ of long foreperiods (and the reverse for the late block). Accordingly, temporal predictions could be built up on the basis of the foreperiod distribution, rather than on the basis of symbolic cues. This redundancy in the source of temporal predictions may have helped overcome interference under dual-task conditions, as attention to cues could prioritize colour over temporal information, which was already afforded by the blocked design. Hence, the amount of resources available in Experiment 1 was sufficiently high to orient attention in time while performing concurrently the WM task. This explanation is supported by the finding that temporal orienting effects in choice-RT tasks are larger when temporal expectancy is manipulated between blocks rather than on a trial-by-trial basis (Correa et al., 2004), which shows that generating a single temporal expectancy across a block of trials regardless of symbolic cues is less demanding than generating a new temporal expectancy after interpreting the meaning of the temporal cue presented on each trial. 
In sum, data from Experiment 1 suggest that temporal orienting based on a blocked design may involve automatic processing, but leave open the question regarding the controlled versus the automatic nature of temporal orienting based on symbolic cueing. To address this issue and to avoid the confounding foreperiod distribution effects mentioned above, in Experiment 2 we manipulated temporal expectancy driven by symbolic cues on a trial-by-trial basis. Assuming that the generation of a new temporal expectancy on each trial would engage a larger amount of controlled processing, thus competing for limited resources with the WM task, we expected to observe reduced temporal orienting effects under the dual-task condition as compared to the single-task condition.

\section{EXPERIMENT 2}

Experiment 2 was conducted to test whether dual-task requirements, which had no effect on temporal orienting based upon a blocked manipulation of expectancies in Experiment 1, may interfere with temporal orienting as participants had to update and shift their temporal expectancy on each trial.

\section{Method}

Participants. Twenty-four new undergraduates from the University of Granada (twentyone females, one left-handed, age range: 18-26 years old) participated in Experiment 2.

Apparatus, stimuli and procedure. The apparatus, stimuli and procedure were the same as those used in Experiment 1 with one critical difference: temporal cues were 
manipulated on a trial-by-trial basis rather than between-blocks of trials. Thus, trials with short lines and trials with long lines (cueing early and late target onsets, respectively) were randomly intermixed within each block.

At the beginning of the experiment, participants were given a short training session to learn the cue-temporal interval contingency, which consisted of four blocks of 16 trials each (100\% valid; cf. Correa et al., 2004). After the training session, half of the participants began with the single-task condition, and half began with the dual-task condition. There were seven blocks for each task condition. The first block of each task condition was considered as practice. Each experimental block consisted of 8 early-cue trials and 8 late-cue trials (cue validity: 75\%). The whole session lasted about 30 minutes.

Design and data analysis. Similarly to Experiment 1, the independent variables were all manipulated within participants, and included Task (single-task versus dual-task), Validity (valid versus invalid), Previous interval (short versus long), and Current interval (short versus long). Participants' RT to respond to the target was the dependent variable.

Data from practice trials, the first trial of each block, trials involving premature responses (i.e., responses before target onset: $3 \%)$, trials with RT below $150 \mathrm{~ms}(0.3 \%)$ and above $1000 \mathrm{~ms}(0.1 \%)$, and trials without responses $(0.6 \%)$ were rejected from the analysis. Mean RTs for each participant and condition were analysed by a repeatedmeasures ANOVA. 
Automatic vs. Controlled temporal preparation

Results

In the WM task, the overall accuracy across participants to the colour memory test was 0.62 .

In the temporal preparation task, the ANOVA revealed slower participants' RT for the dual-task condition than for the single-task condition, which led to a main effect of Task, $F(1,23)=44.89, p<.001$. Crucially, in contrast to Experiment 1 , the Validity effect was modulated by Task condition, as shown by a significant interaction between Task and Validity, $F(1,23)=5.4, p=.029$. Planned comparisons for this interaction revealed that participants' RT was faster for valid trials than for invalid trials only in the single-task condition, $F(1,23)=9.5, p=.005$, but not in the dual-task condition, $F<1$. This finding was also supported by a significant Task x Validity x Current interval interaction, $F(1,23)=5.6, p=.027$, which showed that at the short interval the interaction between Task and Validity was significant, $F(1,23)=7.7, p=.01$, but not at the long interval, $F<1$ (see Figure 4). Specifically, at the short interval temporal orienting effects were significant only in the single-task condition $[31 \mathrm{~ms}, F(1,23)=$ $17.2, p<.001]$ but not in the dual-task condition [ $5 \mathrm{~ms}, F<1]$. At the long interval, no temporal orienting effects were observed in either the single-task or in the dual-task condition, which was expected according to the typical finding of interaction between Validity and Current interval, $F(1,23)=10.16, p=.004$, with a significant Validity effect at the short interval $[13 \mathrm{~ms}, F(1,23)=7.2, p=.01]$ but not at the long interval $[-6$ $\mathrm{ms}, F(1,23)=2.7, p=.1]$. 


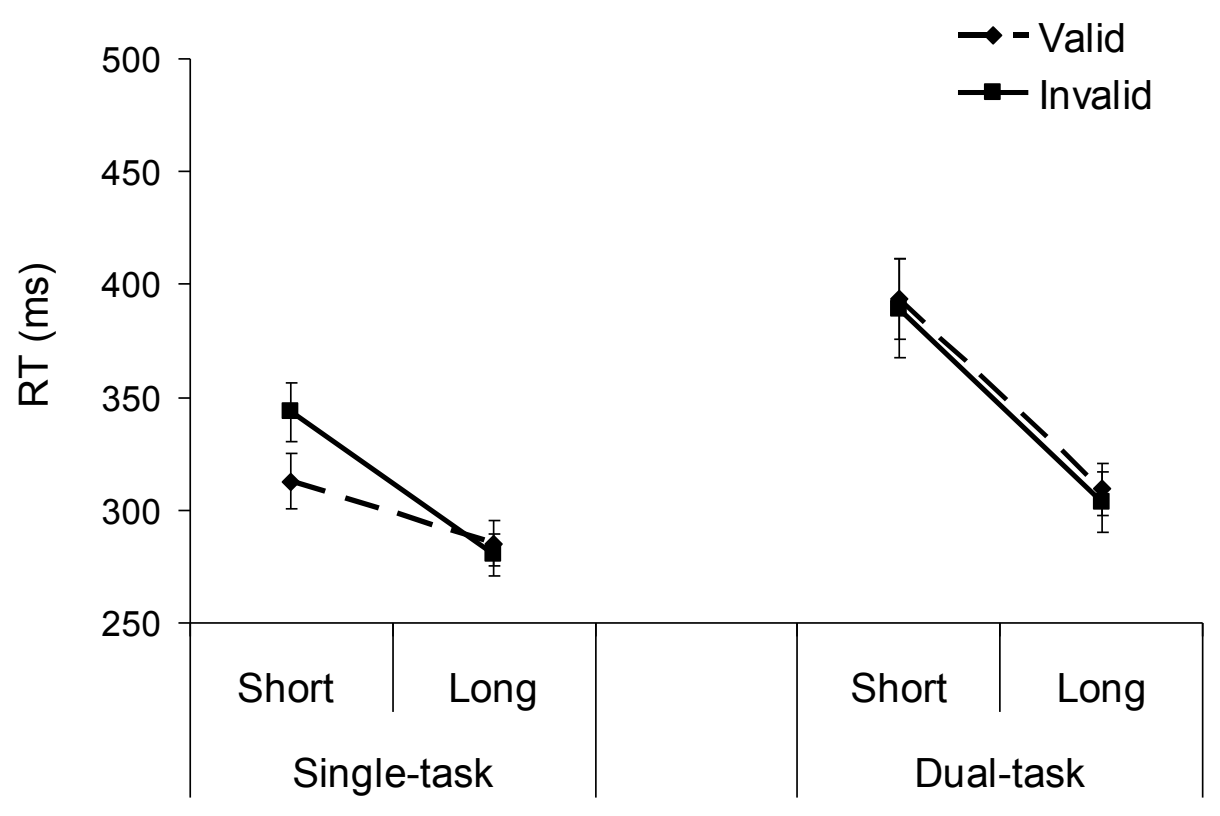

Figure 4. Mean RTs as a function of Task (single-task vs. dual-task), Validity (valid vs. invalid) and Current interval (short vs. long) for Experiment 2 (vertical bars represent standard error of the mean).

The analysis of sequential effects replicated Experiment 1, as both the main effect of Previous interval and the interaction between Previous interval and Current interval were significant, $F(1,23)=57.2, p<.001$ and $F(1,23)=46.9, p<.001$, respectively. The Task x Previous interval interaction was significant, $F(1,23)=5.7, p=$ .026 , showing a greater effect of the previous interval in the dual-task condition, $F(1,23)$ $=38.8, p<.001$, as compared to the single-task condition, $F(1,23)=27.2, p<.001$. The Task $\mathrm{x}$ Previous interval $\mathrm{x}$ Current interval interaction was also significant, $F(1,23)=$ 
$10.7, p=.003$, showing larger sequential effects (RT on Previous long interval minus RT on Previous short interval) at the short interval in the dual-task condition than in the single-task condition, $F(1,23)=13.2, p=.001$ (see Figure 5). It is important to remark that the Previous interval x Current interval interaction was significant in both task conditions, $F(1,23)=59.8, p<.001$, and $F(1,23)=32.3, p<.001$, respectively.

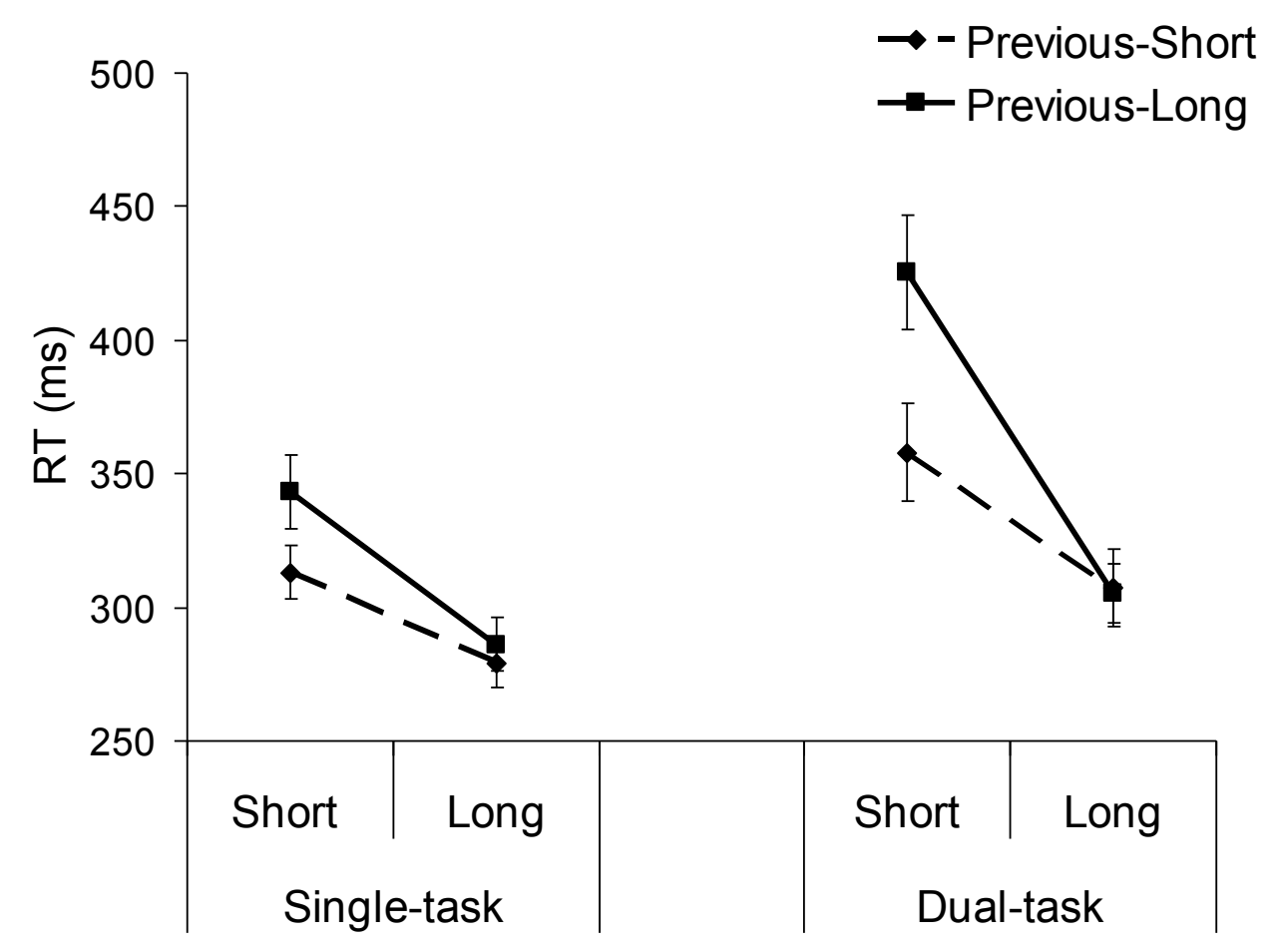

Figure 5. Mean RTs as a function of Task (single-task vs. dual-task), Previous interval (short vs. long) and Current interval (short vs. long) for Experiment 2 (vertical bars represent standard error of the mean). 
Finally, the main effect of Current interval was significant, $F(1,23)=127.9, p$ $<.001$, with participants responding faster at the long interval as compared to the short interval. The significant Task x Current interval interaction was also replicated, $F(1,23)$ $=23.7, p<.001$, with a greater difference in participants' RT between the single-task and the dual-task condition at the short interval $(63 \mathrm{~ms})$ as compared to the long interval (24 ms), although the task effect reached statistical significance in both time intervals $[F(1,23)=53.3, p<.0001, F(1,23)=13.07, p=.001$, for the short and the long interval, respectively].

None of the other terms in the ANOVA was statistically significant except for the Validity $x$ Previous interval interaction, $F(1,23)=7.9, p=.01$, showing larger validity effects after a previous short interval versus a previous long interval $[F(1,23)=$ $6.2, p=.02, F(1,23)=2.4, p=.01$, respectively]

\section{Discussion}

Experiment 2 showed that dual-task requirements impaired temporal orienting effects significantly when temporal cues changed randomly from trial to trial. Participants' RT was indeed faster for valid trials as compared to invalid trials only in the single-task condition. This result suggests that participants were unable to prepare voluntarily when executive resources were depleted by the generation of a new temporal expectancy on each trial and the concurrent WM task.

The Task x Previous interval x Current interval interaction reached statistical significance in this experiment, revealing stronger asymmetric sequential effects in the dual-task condition than in the single-task condition. This finding is therefore convergent with Experiment 1, as sequential effects were neither eliminated nor reduced 
by the secondary task. In the dual-task condition of this experiment, it is worth noting that the increment of sequential effects on one hand, and the disruption of temporal orienting effects on the other hand lend further support to the finding that temporal orienting and sequential effects involve dissociable processes (Correa et al., 2004; Correa et al., 2006; Los \& Heslenfeld, 2005; Los \& Van den Heuvel, 2001). Attention was not necessary for the occurrence of sequential effects since they were present by default regardless of voluntary effort, and, as highlighted by the results of Experiment 2, they were even larger in conditions where attention was divided between two demanding tasks (cf. Los \& Van den Heuvel, 2001). On the contrary, temporal orienting (manipulated within-blocks of trials) required attentional control, so that when participants were forced to divert their attention from the temporal preparation task by simultaneously performing the WM task, temporal orienting effects were completely abolished.

The results of Experiment 2 confirmed that the presence of a secondary task interfered with participants' ability to orient attention in the temporal domain. However, this latter finding could also be explained by interference between the interpretation of the temporal cue as signalling an early and late target onset and the processing of cue colour information for the memory task. Specifically, the absence of temporal orienting effects in Experiment 2 might be due to the fact that participants neglected to process the meaning of the temporal cue because they were engaged in updating the colour information. This possibility was tested in Experiment 3. 


\section{EXPERIMENT 3}

Experiment 3 was similar to Experiment 2 except in one critical aspect: the temporal cues were always displayed in gray in order to avoid any possible confound with the WM task. WM was loaded at the beginning of each trial by presenting participants with one of three coloured (red, green or blue) stars. As in Experiments 1 and 2, we manipulated WM demands by having participants remember and report the final count of each colour at the end of every block. However, differently from Experiment 2, this design ensured that the updating of the count in WM would not interfere with the processing and interpretation of the temporal cues. On the premises that temporal orienting relies on controlled processing, we expected Experiment 3 to replicate the main finding of Experiment 2, that is, a significant interaction between Task and Validity.

\section{Method}

Participants. Twenty-eight new undergraduates from the University of Granada (twenty-three females, five left-handed, age range: 18-46 years old) participated in Experiment 3.

Apparatus, stimuli and procedure. Everything was the same as in Experiment 2 except for the following: 1) the temporal cues were always displayed in gray; and 2) the memory stimuli consisted of three coloured (red, green and blue) stars $\left(1.31^{\circ} \times 1.01^{\circ}\right.$ visual angle). In each trial a blank screen was presented for $50 \mathrm{~ms}$, followed by a 200ms period which was used to display the coloured star. After the presentation of the 
Automatic vs. Controlled temporal preparation

memory stimulus, there was a random interval ranging from 500 and $1000 \mathrm{~ms}$ before the presentation of the temporal cue.

Design and data analysis. The independent variables were all manipulated within participants, and included Task (single-task versus dual-task), Validity (valid versus invalid), Previous interval (short versus long), and Current interval (short versus long). Participants' RT to respond to the target was the dependent variable.

Data from practice trials, the first trial of each block, trials involving premature responses (i.e., responses before target onset: $1.2 \%$ ), trials with RT below $150 \mathrm{~ms}$ $(0.2 \%)$ and above $1000 \mathrm{~ms}(0.2 \%)$, and trials without responses $(0.9 \%)$ were rejected from the analysis. Mean RTs for each participant and condition were analysed by a repeated-measures ANOVA.

Results

In the WM task, the overall accuracy across participants to the colour memory test was 0.64 .

In the temporal preparation task, the ANOVA replicated the significant effects of Task, $F(1,27)=40.6, p<.001$, Validity, $F(1,27)=17.44, p<.001$, and the interaction between Validity and Current interval, $F(1,27)=27.82, p<.001$. Most relevant, there was a significant interaction between Task and Validity, $F(1,27)=4.8$, $p=.03$, with the validity effect being significant only in the single-task condition (20 $\mathrm{ms}, F(1,27)=30.41, p<.001)$, but not in the dual-task condition $(5 \mathrm{~ms}, F<1)$.

Although the Task x Validity x Current interval interaction was not significant, $F(1,27)=1.65, p=.2$, hypothesis-driven planned comparisons (see Rutherford, 2001; 
Keppel \& Zedeck, 1989) at the short interval revealed that the validity effect was half size smaller in the dual-task condition than in the single-task condition (21 ms vs. 43 ms), Task x Validity: $F(1,27)=4.03, p=.05$ (this interaction was not significant at the long interval, $p=.2$ ). It is important to note that the validity effect at the short interval was significant for both the single-task, $F(1,27)=36.24, p<.001$, and the dual-task condition, $F(1,27)=5.58, p=.02($ see Figure 6$)$.

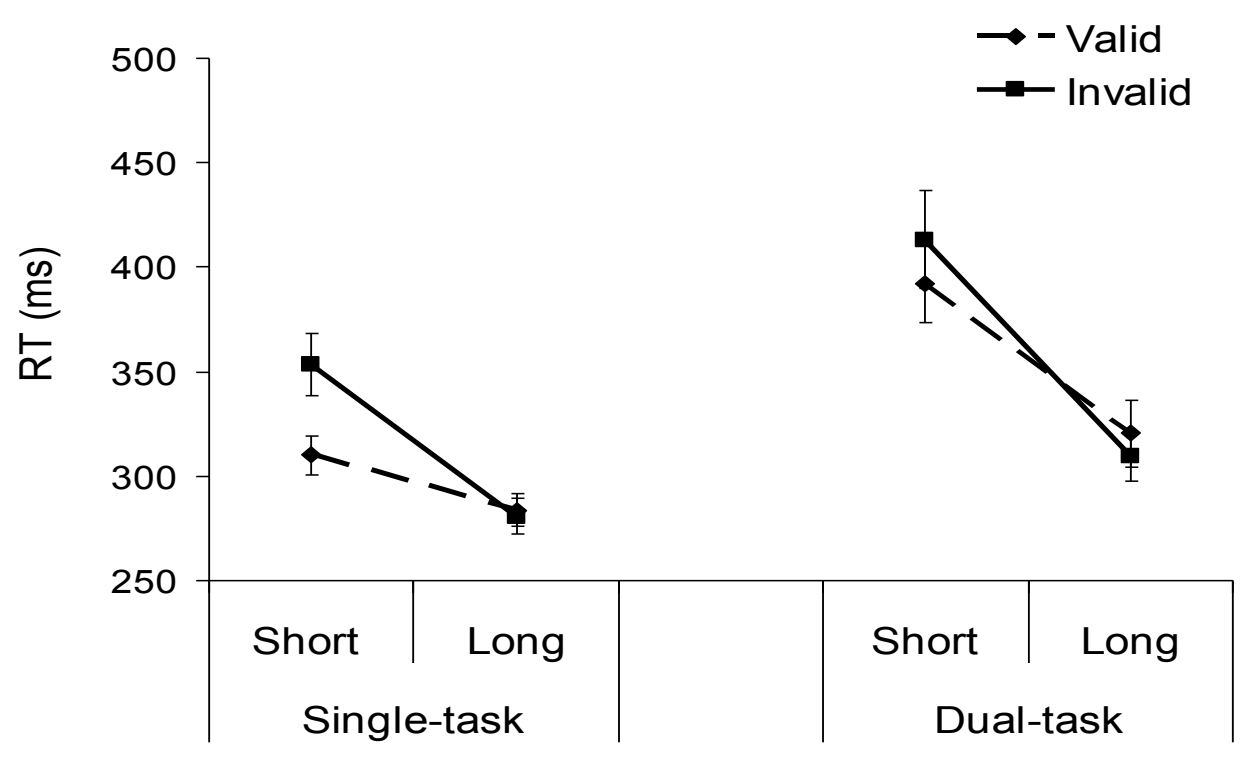

Figure 6. Mean RTs as a function of Task (single-task vs. dual-task), Validity (valid vs. invalid) and Current interval (short vs. long) for Experiment 3 (vertical bars represent standard error of the mean).

The analysis of sequential effects replicated Experiment 1, with significant effects of Current interval, $F(1,27)=156.2, p<.001$, Previous interval, $F(1,27)=$ 75.04, $p<.001$, and interaction between Previous interval and Current interval, $F(1,27)$ $=46.55, p<.001$. The critical finding was that sequential effects were not attenuated by 
dual-task demands $(F \mathrm{~s}<1$ for both Task $\mathrm{x}$ Previous interval and Task $\mathrm{x}$ Previous interval $\mathrm{x}$ Current interval interactions; see Figure 7). None of the other terms in the ANOVA was statistically significant except for the Task x Current interval interaction, $F(1,27)$ $=16.7, p<.001$.

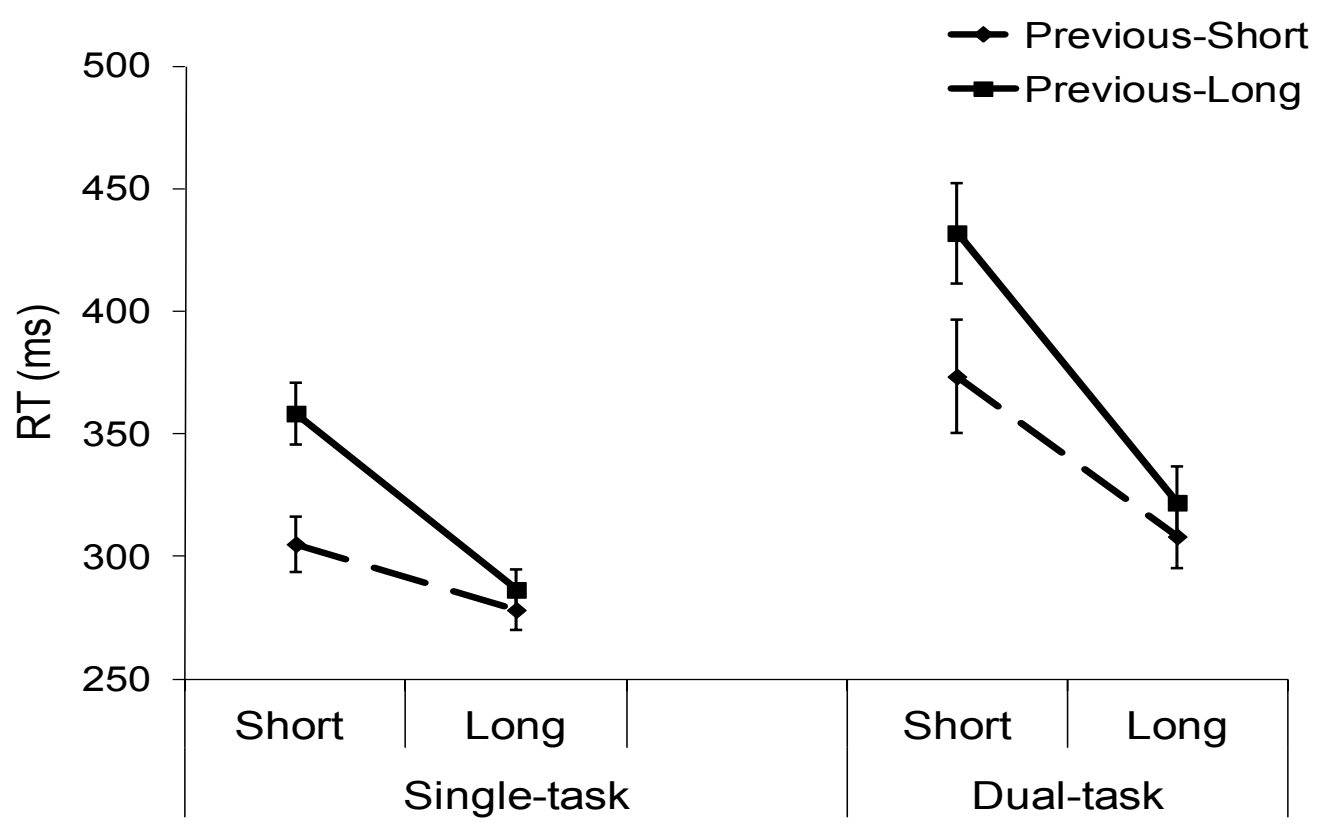

Figure 7. Mean RTs as a function of Task (single-task vs. dual-task), Previous interval (short vs. long) and Current interval (short vs. long) for Experiment 3 (vertical bars represent standard error of the mean).

\section{Discussion}

The results from Experiment 3 showed that sequential effects were not reduced by the secondary memory task, a finding that supports their automatic nature. In contrast, temporal orienting effects were larger in the single-task condition as compared to the dual-task condition. This finding renders unlikely the possibility that the absence of temporal orienting effects in Experiment 2 was simply due to interference between 
the updating of the count in WM and the interpretation of the temporal cues, because in the present experiment the temporal preparation task and the WM task did not share the same stimulus. In this regard, it is important to note that the results of Experiments 2 and 3 differed in one critical aspect. Namely, the validity effect at the short interval was completely abolished by dual-task demands in Experiment 2, while it was significant, although reduced, with respect to the single task condition in Experiment 3. This finding confirmed that in Experiment 3 participants were engaged in processing the meaning of the temporal cue, which suggests that the reduction of the validity effect was due to dual-task interference and the fact that temporal orienting relies on controlled processing.

\section{General Discussion}

In three experiments, a dual-task approach was used to directly test the nature of the processes involved in temporal preparation. The logic of our design was based on the criterion that controlled processing would be reduced by a demanding secondary task, whereas automatic processing would not be attenuated by such a dual-task context (e.g., Logan, 1979; Posner \& Snyder, 1975). The results of Experiments 1-3 showed that temporal orienting and sequential effects could be behaviourally dissociated by using a dual-task paradigm. The current data provide direct support to the idea that both controlled and automatic processes may contribute independently to the development of temporal preparation (Correa, 2010; Los and Van den Heuvel, 2001).

Temporal orienting effects were completely abolished under dual-task conditions in Experiment 2, where the same stimulus (the temporal cue) elicited updating of both WM and temporal expectancy on a trial-by-trial basis. Separating the 
memory cue from the temporal cue in Experiment 3 did not completely eliminate temporal orienting effects on short-interval dual-task trials, proving that participants were actually processing the meaning of the temporal cue. However, despite encoding of temporal cues was enabled in this experiment, temporal orienting effects were significantly reduced in the dual-task condition as compared to the single-task condition. Taken together, these findings suggest a gradation of the competition for attentional resources between temporal preparation and WM tasks across the three experiments reported in the present manuscript (cf. Logan, 1978, 1979). As Figure 8 shows, maximal competition would have occurred in Experiment 2 (updating of WM and temporal expectancy took place simultaneously), followed by Experiment 3 (the two updating processes were separated in time), and Experiment 1 (only updating of WM but not of temporal expectancy was required on each trial).

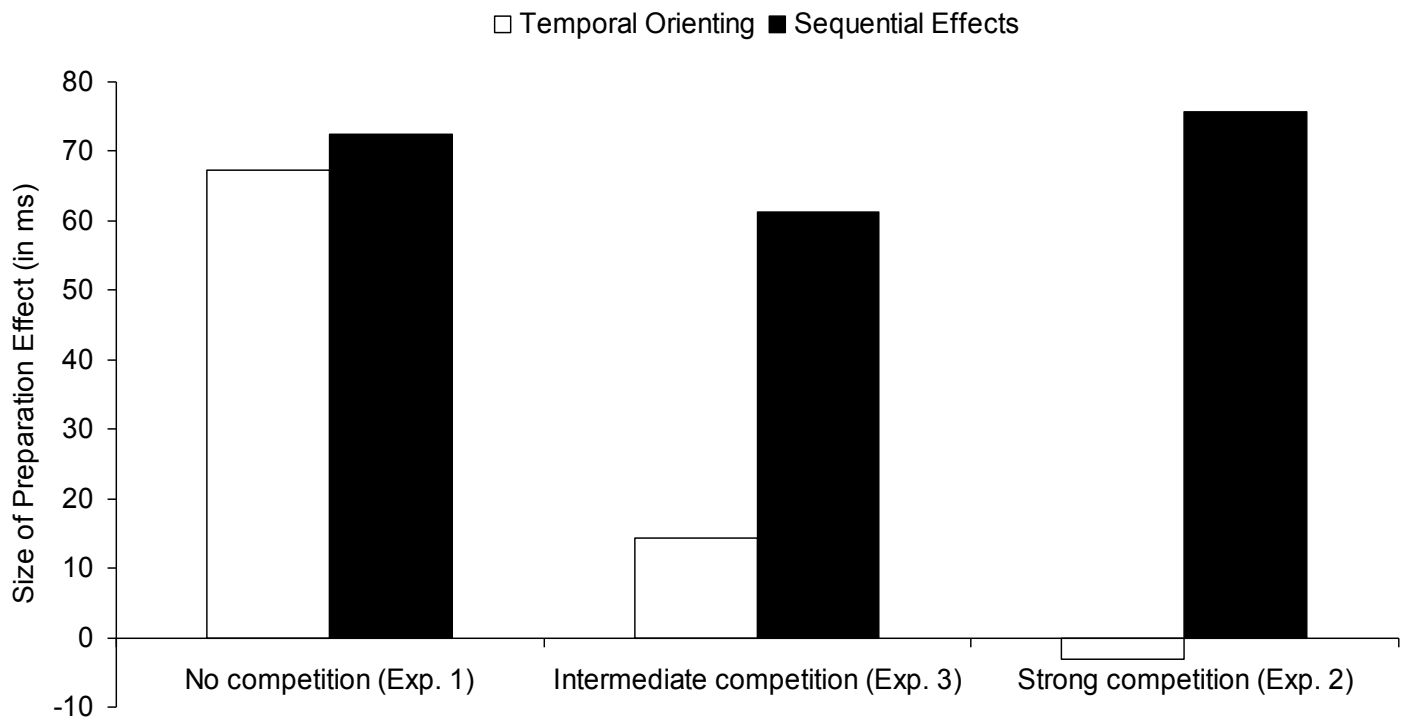

Figure 8. Competition between updating of working memory versus temporal expectancy for temporal orienting and sequential effects across the three experiments. The size of the preparation effects was computed for both temporal orienting (RTinvalid minus RT-valid) and sequential effects (RT-previous long minus RT-previous short) at the short interval. Data are from the dual-task condition. 
This pattern of results supports the idea that the distinction between controlled and automatic processing should be perceived as a continuum rather than a strict dichotomy. Such a view is in agreement with earlier studies showing that controlled processing may develop into automatic processing after practice at a task (Posner \& Snyder, 1975; Shiffrin \& Schneider, 1977), and that automatic processing is not cognitively impenetrable or "encapsulated" (cf. Fodor, 1983) since it may be susceptible of modulation by controlled factors (see Ruz \& Lupiáñez, 2002, for an example on the attentional capture literature). Our data extend these observations in the temporal preparation domain by showing that the balance between controlled and automatic processing in temporal orienting of attention can be affected by several factors, such as the way in which temporal information is provided.

To sum up, the results of the three experiments presented here provide the first behavioural evidence that a different amount of executive resources may be recruited by instructing participants to shift their temporal expectancy across trials as compared to focus their attention along the whole block (cf. Correa et al., 2004, 2006). This is in line with research showing that temporal orienting involves different brain areas in betweenblocks as compared to within-blocks manipulation of temporal expectancies. Namely, a recent neuropsychological study has found a clear lateralization of temporal orienting effects in the context of a blocked-design in the right prefrontal cortex, while no temporal orienting deficit was observed in patients with left frontal lesions (Triviño et al., 2010). Conversely, another functional MRI study has reported the involvement of left prefrontal structures when temporal cues were manipulated on a trial-by-trial basis, suggesting that updating and shifting the temporal information provided by the cue may engage different neural circuits (Coull \& Nobre, 1998). 
In contrast to temporal orienting, sequential effects survived to dual-task interference in Experiments 1-3, as they were neither eliminated nor reduced by concurrent task demands. This finding supports the dissociation between temporal orienting and sequential effects and reinforces the hypothesis that sequential effects are generated by automatic processing (see Correa et al., 2004; Correa et al., 2006; Los \& Heslenfeld, 2005; Los \& Van den Heuvel, 2001). In line with this latter idea, an interesting finding of Experiment 2 was the increased magnitude of sequential effects under dual-task relative to single-task conditions. It would appear that the high attentional demands and competition between temporal orienting and memory tasks in Experiment 2 resulted in an enhancement of the automatic processing responsible for sequential effects. Although plausible, however, such idea deserves further investigation as a pattern of enhanced sequential effects under dual-task conditions was observed only in Experiment 2. Moreover, a dual-task study by Van Lambalgen and Los (2008) reported that extra processing demands interfered with sequential effects by reducing, instead of enhancing, their asymmetry. Future research shall address the discrepancy between Van Lambalgen and Los's study (2008) and the present findings by testing sequential effects across different secondary tasks, in order to explore under which conditions they may interact or not with concurrent task demands.

In conclusion, the present study dissociated the involvement of automatic and controlled processes in temporal preparation, and proved for the first time the effectiveness of dual-task methodology in investigating the nature of both temporal orienting and sequential effects. A challenge for future research would be to specify the 
boundary conditions determining the expression of automatic and controlled temporal preparation processing. 
Automatic vs. Controlled temporal preparation

\section{REFERENCES}

Brown, S. W. (2006). Timing and executive function: bidirectional interference between concurrent temporal production and randomization tasks. Memory and Cognition, 34(7), 1464-1471.

Correa, A. (2010). Enhancing behavioural performance by visual temporal orienting. In A.C. Nobre and J.T. Coull (Eds.). Attention and Time (p. 357-370). Oxford University Press.

Correa, A., Lupiáñez, J., Milliken, B., \& Tudela, P. (2004). Endogenous temporal orienting of attention in detection and discrimination tasks. Perception and Psychophysics, 66(2), 264-278.

Correa, A., Lupiáñez, J., \& Tudela, P. (2006). The attentional mechanism of temporal orienting: Determinants and attributes. Experimental Brain Research, 169(1), $58-68$.

Coull, J. T., \& Nobre, A. C. (1998). Where and when to pay attention: The neural systems for directing attention to spatial locations and to time intervals as revealed by both PET and fMRI. Journal of Neuroscience, 18(18), 7426-7435.

Drazin, D. H. (1961). Effects of foreperiod, foreperiod variability, and probability of stimulus occurrence on simple reaction time. Journal of Experimental Psychology, 62, 43-50.

Fodor, J. A. (1983). Modularity of mind : an essay on faculty psychology. Cambridge: MIT Press.

Fortin, C., \& Breton, R. (1995). Temporal interval production and processing in working memory. Perception \& Psychophysics, 57, 203-215. 
Karlin, L. (1959). Reaction time as a function of foreperiod duration and variability. Journal of Experimental Psychology, 58, 185-191.

Keppel, G., \& Zedeck, S. (1989). Data analysis for research designs: Analysis of variance and multiple regression/correlation approaches. Freeman, New York.

Lewis, P. A., \& Miall, R. C. (2006). Remembering the time: a continuous clock. Trends in Cognitive Sciences, 10(9), 401-406.

Logan, G. D. (1978). Attention in character-classification tasks: Evidence for the automaticity of component stages. Journal of Experimental Psychology: General, 107, 32-63.

Logan, G. D. (1979). On the use of a concurrent memory load to measure attention and automaticity. Journal of Experimental Psychology: Human Perception and Performance, 5, 189-207.

Los, S. A. (1996). On the origin of mixing costs: Exploring information processing in pure and mixed blocks of trials. Acta Psychologica, 94, 145-188.

Los, S. A., \& Heslenfeld, D. J. (2005). Intentional and unintentional contributions to nonspecific preparation: Electrophysiological evidence. Journal of Experimental Psychology: General, 134, 52-72.

Los, S. A., \& Van den Heuvel, C. E. (2001). Intentional and unintentional contributions to nonspecific preparation during reaction time foreperiods. Journal of Experimental Psychology: Human Perception and Performance, 27, 370-386.

Nobre, A. C. (2001). Orienting attention to instants in time. Neuropsychologia, 39, $1317-1328$.

Nobre, A. C., Correa, A., \& Coull, J. T. (2007). The hazards of time. Current Opinion in Neurobiology, 17, 1-6. 
Automatic vs. Controlled temporal preparation

Posner, M. I., \& Snyder, C. R. R. (1975). Attention and cognitive control. In R. Solso (Ed.), Information processing and cognition: The Loyola Symposium (pp. 5585). Hillsdale, NJ: Lawrence Erlbaum.

Posner, M. I., Snyder, C. R. R., \& Davidson, B. J. (1980). Attention and the detection of signals. Journal of Experimental Psychology: General, 109, 160-174.

Rutherford, A. (2001). Introducing ANOVA and ANCOVA: a GLM approach.

Ruz, M., \& Lupiáñez, J. (2002). A review of attentional capture. Psicológica, 23, 283309.

Schneider, W., Eschman, A., \& Zuccolotto, A. (2002). E-Prime user's guide. Pittsburgh: Psychology Software Tools Inc.

Shiffrin, R. M., \& Schneider, W. (1977). Controlled and automatic human information processing: II. Perceptual learning, automatic attending and a general theory. Psychological Review, 84, 127-190.

Steinborn, M. B., Rolke, B., Bratzke, D., \& Ulrich, R. (2008). Sequential effects within a short foreperiod context: evidence for the conditioning account of temporal preparation. Acta Psychologica (Amst), 129(2), 297-307.

Triviño, M., Correa, A., Arnedo, M., \& Lupiáñez, J. (2010). Temporal orienting deficit after prefrontal damage. Brain, 133, 1173-1185.

Vallesi, A., \& Shallice, T. (2007). Developmental dissociations of preparation over time: deconstructing the variable foreperiod phenomena. Journal of Experimental Psychology: Human Perception and Performance, 33(6), 13771388.

Van Lambalgen, R. M., \& Los, S. A. (2008). The role of attention in nonspecific preparation. In B. C. Love, K. McRae \& V. M. Sloutsky (Eds.), Proceedings of 
the 30th Annual Conference of the Cognitive Science Society (pp. 1525-1530). Austin, TX: Cognitive Science Society.

Woodrow, H. (1914). The measurement of attention. Psychological Monographs, 17, 1158. 
Automatic vs. Controlled temporal preparation

\section{ACKNOWLEDGEMENTS}

Financial support to this research was provided by a FPI predoctoral grant from the Spanish Ministerio de Ciencia e Innovación (MICINN) to Mariagrazia Capizzi, and by the Ramón y Cajal programme (RYC-2007-00296) and the Junta de Andalucía by the P09-HUM-5422 and P-07-SEJ3299 grants (Proyectos de Excelencia) to Ángel Correa and Daniel Sanabria, and by the SEJ-6414 (Proyecto de Excelencia, Junta de Andalucía), SEJ2007-63645, SEJ2007-63247, PSI2010-19655, PSI2010-15399 (I+D) and CSD2008-00048 (Consolider) grants from the MICINN to Daniel Sanabria and Ángel Correa. We thank Sander Los and two anonymous reviewers for their useful comments on earlier versions of the manuscript. 


\section{FOOTNOTES}

1. One might argue that participants guessed the outcome of the secondary task as the expected value of the total number of colours in each block was $5.33(16 / 3)$, which would led them to respond " 5 " systematically. This guessing strategy seems unlikely as participants were not informed or aware of the total number of trials in each block. Moreover, if participants were following this guessing strategy in a consistent way, we would expect no effects of the dual-task manipulation. In contrast, we found significant effects of dual-task interference on RT in the temporal preparation task in all the three experiments. This result confirmed that the dual-task manipulation was effective, that is, participants were actually engaged rather than neglecting the secondary task.

2. It is possible that the finding of larger dual-task interference at the short time interval as compared to the long time interval was a consequence of the experimental design used in our study. Since WM demands were placed into the temporal cue, one might argue that less time was available for memory updating and rehearsal of the colours at the short interval than at the long interval, thus explaining the Task by Current interval interaction. Although interesting, this aspect is beyond the scope of the present work (but see Van Lambalgen and Los, 2008, for empirical evidence on this issue), which was focused on the consequence of WM demands upon temporal orienting and sequential effects. 